Radial and Nonradial Pulsations as Probes of Stellar Physics

ASP Conference Series, Vol. 259, 2002

C. Aerts, T.R. Bedding, \& J. Christensen-Dalsgaard, eds.

\title{
RR Lyrae Variables in Local Group Galaxies: M31 ${ }^{1}$
}

\author{
G. Clementini, L. Federici, C. Cacciari, M. Bellazzini
}

Osservatorio Astronomico di Bologna, Via Ranzani 1, I-40127 Bologna, Italy, e-mail: gisella@bo astro.it

C. Corsi

Osservatorio Astronomico di Roma, Via di Frascati 33, I-00040 Monte Porzio Catone, Italy

\section{H.A. Smith}

Department of Physics and Astronomy, Michigan State University, East Lansing, $M I$ 48824-1116

\begin{abstract}
Candidate RR Lyrae variables have been identified for the first time in four globular clusters of the Andromeda galaxy (M31). Using HST-WFPC2 archive observations we have found 2, 4, 11 and 8 RR Lyrae variables of both $a b$ and $c$ Bailey types in G11, G33, G64 and G322 (Sargent et al., 1977), respectively. These numbers are quite consistent with the horizontal branch morphology exhibited by the four clusters.
\end{abstract}

\section{Introduction}

RR Lyraes are a common type of variables in almost all of the Local Group galaxies. Their identification and study can provide fundamental insights into the distance scale, the stellar content, and the formation process of the hosting galaxy. Because of its large distance, a few $R R$ Lyrae variables have been identified so far only in the field of M31 (Pritchet \& van den Bergh, 1987). Exploiting the high spatial resolution of the Hubble Space Telescope (HST) we were able to identify candidate RR Lyrae variables in four of the M31 globular clusters.

\section{Observational database and variable search results}

HST color magnitude diagrams (CMDs) reaching one magnitude below the HB are at present available for more than a dozen of the M31 globular clusters. Among them we have selected four clusters in reasonably uncrowded regions of the galaxy, that had well defined CMDs and populous enough HBs. The HST ( $\mathrm{F} 555 \mathrm{~W}$ and $\mathrm{F} 814 \mathrm{~W}$ ) frames for the selected clusters were reduced individually

\footnotetext{
'Based on observations made with the NASA/ESA Hubble Space Telescope, obtained from the data archive at the Space Telescope Science Institute. STScI is operated by the Association of Universities for Research in Astronomy, Inc. under NASA contract NAS 5-26555.
} 


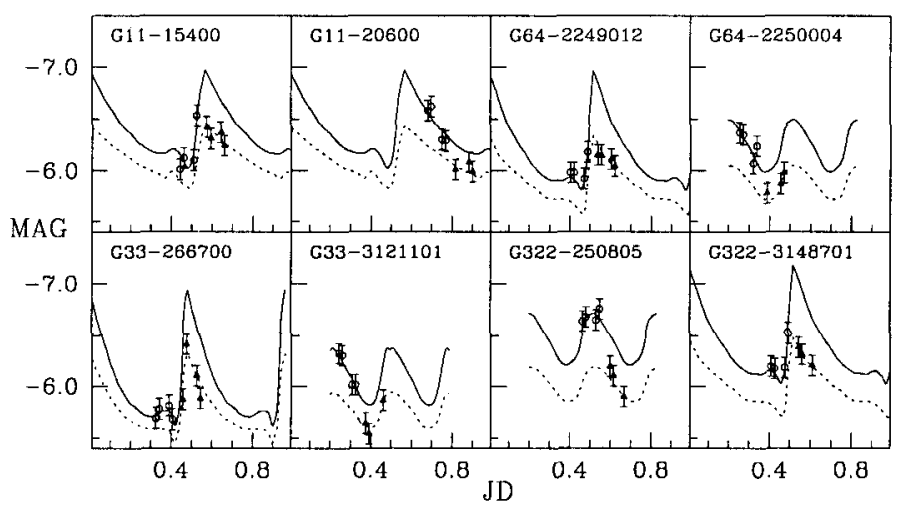

Figure 1. Examples of HST V (circles) and I (triangles) data fitting to RR Lyrae template V (solid lines) and I (dotted lines) light curves. The vertical scale is magnitudes with an arbitrary zero-point.

using the ROMAFOT reduction package (Buonanno et al., 1983). Variability search was then performed on the basis of the scatter of the individual photometric measures with respect to the average value. We considered only objects with r.m.s. deviations larger than 0.3 mag., i.e. $3 \sigma$ from the average r.m.s. value. The sequences of individual measurements displayed as a function of the Julian Day of observation were then compared with template $\mathrm{V}$ and I light curves of RR Lyrae variables in the globular cluster $\mathrm{M} 3([\mathrm{Fe} / \mathrm{H}] \sim-1.5$ and $\mathrm{E}(\mathrm{B}-\mathrm{V})=0.0$, Carretta et al., 1998). Examples of these comparisons are shown in Fig. 1.

Using this procedure we were able to derive an indication of variability for 2 , 4, 11 and 8 RR Lyrae candidates in G11, G33, G64 and G322, respectively (see also Clementini et al., 2001). Assuming $(m-M)_{0}=24.43$ for M31 (Freedman \& Madore, 1990), the apparent luminosity of the RR Lyrae candidates leads to a rather "bright" absolute magnitude, in better agreement with the "long" distance scale.

\section{References}

Buonanno, R., Buscema, G., Corsi, C.E., Ferraro, I., \& Iannicola, G. 1983, A\&A, 126,278

Carretta, E., Cacciari, C., Ferraro, F.R., Fusi Pecci, \& Tessicini, G. 1998, MNRAS, 298, 1005

Clementini, G., Federici, L., Corsi, C.E., Cacciari, C., Bellazzini, M., Smith, H.A. 2001, ApJL, 559, 109

Freedman, W., \& Madore, B. 1990, ApJ, 365, 186

Pritchet, C.J., \& van den Bergh, S. 1987, ApJ, 316, 517

Sargent, W.L.W., Kowal, C.T., Hartwick, F.D.A., \& van den Bergh, S. 1977, $\mathrm{AJ}, 82,947$ 